\title{
AVALIAÇÃO ERGONÔMICA DO POSTO DE TRABALHO DE MODELOS DE "FORWARDER" E "SKIDDER"1
}

\author{
Gustavo Fontana² e Fernando Seixas ${ }^{3}$
}

\begin{abstract}
RESUMO - O processo de mecanização florestal no Brasil envolve, muitas vezes, o uso de máquinas adaptadas ou importadas de países com operadores com diferentes características antropométricas. O presente trabalho teve por objetivo realizar a avaliação ergonômica da cabine de seis modelos de máquinas florestais utilizadas na extração de madeira (quatro "forwarders" e dois "skidders"), quanto ao posicionamento de comandos e instrumentos, com base nas características antropométricas de um conjunto de operadores brasileiros. Foram estudados os seguintes tratores: "forwarders" Timberjack modelos 1210B e 1710D, Valmet 890.2 e Volvo A25C, além dos "skidders" Caterpillar 545 e Tigercat 630 B. Para avaliação do posicionamento dos órgãos de comando foram determinadas as distâncias destes mesmos a partir do Ponto de Referência do Assento nas três dimensões (x, y e z), além de uma avaliação qualitativa com os operadores dos tratores florestais em estudo, que, através de notas, manifestaram sua satisfação com relação à localização dos comandos e outras variáveis ergonômicas. A avaliação antropométrica dos operadores foi feita por duas medidas, uma em pé e outra por meio de uma cadeira especial desenvolvida pela Fundacentro, obtendo-se, a partir dela, as áreas de máximo e ótimo acesso quanto à localização dos órgãos de comando. Concluiu-se que a melhor máquina, quanto ao posicionamento de comandos, foi o "forwarder" Valmet 890.2, seguido pelo "skidder" Caterpillar 545, sendo os únicos tratores que apresentaram mais da metade dos comandos bem posicionados, 66,7 e 54,5\%, respectivamente. Os resultados deste estudo demonstraram um projeto ergonômico da disposição de comandos nas cabines dos tratores florestais não muito favorável ao conjunto de operadores brasileiros analisados.
\end{abstract}

Palavras-chave: Antropometria, ergonomia, tratores florestais e colheita.

\section{ERGONOMIC EVALUATION OF THE WORKSTATION OF FORWARDER AND SKIDDER MODELS}

\begin{abstract}
The logging mechanization process in Brazil means, most of the time, the use of adapted or imported machines from countries where the operators have different anthropometric characteristics. The objective of this work was the cab evaluation of four forwarders and two skidders, considering the position of commands and instruments and the operator visual area, based on the anthropometrics characteristics of a sample of Brazilians operators. The following machines were studied: forwarders Timberjack models $1210 B$ and $1710 D$, Valmet 890.2 and Volvo A25C, and also the skidders Caterpillar 545 and Tigercat 630 $B$. The command position was determined in three dimensions ( $x$, $y$ and $z$ ), considering the operator's seat reference point as the origin. A qualitative evaluation with the operators was also carried out, showing their satisfaction with several ergonomic factors related with the forest machines. The operator anthropometric evaluation was performed using a special chair developed by the Fundacentro, resulting in areas of maximum and excellent access of command position. The machine presenting the best command positions, according to the biotype of the operator sample, was the forwarder Valmet 890.2, followed by the skidder Caterpillar 545 , being the only tractors showing more than half of commands well positioned, $66.7 \%$ and $54.5 \%$ respectively. The results of this study demonstrated that, in terms of command position, the ergonomic design of these machines was not very favorable to the set of analyzed Brazilians operators.
\end{abstract}

Keywords: Anthropometry, ergonomy, forest tractors and harvesting.

\footnotetext{
${ }^{1}$ Recebido em 10.04.2006 e aceito para publicação em 15.12.2006.

${ }^{2}$ Departamento de Engenharia Rural, ESALQ/USP, Piracicaba-SP. (16) 9112-9590. E-mail: <agri_fontana@ yahoo.com.br>.

${ }^{3}$ Departamento de Ciências Florestais, ESALQ/USP, Piracicaba-SP. E-mail:<fseixas@esalq.usp.br>.
} 


\section{INTRODUÇÃO}

A mecanização florestal, na maioria das vezes, utiliza máquinas adaptadas ou importadas de países com diferentes condições climáticas e características antropométricas dos operadores. O custo elevado dessas máquinas exige o máximo de aproveitamento de todas as suas funções durante a execução contínua das tarefas a elas atribuídas, bem como demanda estudos no sentido de adequá-las às condições de trabalho no Brasil.

A principal atividade dos operadores dessas máquinas é realizada no posto de condução na cabine, daí a importância da aplicação de critérios ergonômicos que permitam estabelecer a correta adaptação dos componentes do sistema homem-máquina. Para isso, é preciso levar em conta as características dos operadores e do trabalho que se realiza, alcançando, desse modo, maior eficiência produtiva, assim como maior grau de conforto e segurança na tarefa, proporcionando-se uma melhoria das condições de trabalho. A ergonomia tem contribuído significativamente para a melhoria das condições de trabalho humano. Entretanto, na maioria dos países em desenvolvimento é um conceito relativamente novo, e essa contribuição ainda é pequena, em razão do baixo número de estudos e da restrita divulgação dos seus benefícios (MINETTE et al., 1998).

Silva et al. (2003) realizou uma avaliação ergonômica, com base no manual "Ergonomic Guidelines for Forest Machines” (SKOGFORSK, 1999), de um “feller-buncher”. Concluiu que o "feller-buncher" apresentava alto grau de segurança ativa e passiva. Verificou-se também a necessidade de algum ajuste das condições do espaço de trabalho ao operador brasileiro, em virtude de suas características antropométricas serem diferentes das dos europeus, para os quais a máquina foi desenhada.

Lima et al. (2005) realizaram a avaliação ergonômica nos tratores florestais "feller-buncher" (411EX HidroAx) e "skidder" (CAT 525), com o objetivo de avaliar as dimensões de acesso, assento, comandos, campo visual, condições térmicas, vibração, nível de ruído e inclinação que permite a estabilidade longitudinal e transversal para o tráfego, concluindo que o "skidder" teve seu acesso ao posto do operador classificado como bom e o "feller-buncher" como médio. Os assentos nas duas máquinas foram considerados como bons na avaliação qualitativa. Para o posto do operador, o "skidder" teve melhor classificação, comparando o espaço livre de 55,0\% contra 30,0\% do "feller-buncher".
A norma que trata de ergonomia no Brasil é a NR17, do Ministério do Trabalho e Emprego, publicada em 1978 e modernizada em 1990. Essa norma tem por objetivo estabelecer parâmetros que permitam a adaptação das condições de trabalho às características psicofisiológicas dos trabalhadores, de modo a proporcionar o máximo conforto, segurança e desempenho. Entretanto, não há indicação para avaliação ergonômica de máquinas florestais, conforme já existente nos países escandinavos (FONTANA, 2005).

No caso de máquinas de extração de madeira, segundo Makkonen (1989) o "forwarder" é considerado a máquina mais bem projetada do ponto de vista ergonômico e a sua operação como menos cansativa, favorecendo a sua adoção em países com legislação trabalhista mais rigorosa.

Nesse contexto, o presente trabalho teve por objetivo realizar a avaliação ergonômica comparativa da cabine de seis modelos de máquinas florestais utilizadas na extração de madeira (quatro "forwarders" e dois "skidders"), quanto ao posicionamento de comandos e instrumentos, considerando-se as características antropométricas de um conjunto de operadores brasileiros analisados e concluir pela sua adaptação ou não ao biótipo do nosso trabalhador.

\section{MATERIAL E MÉTODOS}

\subsection{Máquinas}

Foram utilizados neste trabalho quatro modelos e três marcas de "forwarders" e dois modelos e marcas de "skidders", cujas características são descritas nos Quadros 1 e 2, respectivamente. Os dados foram coletados em duas empresas florestais, localizadas nos Municípios de Mogi-Guaçu e Lençóis Paulista, no Estado de São Paulo.

\subsection{Dispositivo para simulação do ponto de referência do assento (SIP)}

A avaliação ergonômica dos tratores foi realizada por meio de medidas a partir do ponto de referência do assento ("Seat Index Point" - SIP), que, de acordo com a norma NBR NM-ISO 5353:1999, "pode ser considerado para fins de projeto do local de trabalho do operador, ser equivalente à intersecção do plano vertical central que passa pela linha de centro do assento no eixo de rotação teórico entre o tronco e coxas humanos". 
Quadro 1 - Características técnicas dos modelos de "forwarders" Table 1 - Characteristics of the forwarders

\begin{tabular}{lcccc}
\hline Características & \multicolumn{2}{c}{ Timberjack } & Valmet & Volvo \\
\cline { 2 - 5 } & $1210 \mathrm{~B}$ & $1710 \mathrm{D}$ & 890.2 & A25C \\
\hline Potência kW - (cv) & $128-(174)$ & $215-(292)$ & $151-(205)$ & $190-(258)$ \\
Especificação do Motor & Perkins 1006 & John Deere 6018HT & Valmet 634 DWIE & Volvo TD73KCE \\
Massa (kg) & 15000 & 17500 & 16800 & 16000 \\
Carga útil (kg) & 12000 & 17000 & 18000 & 21000 \\
Originário & EUA & EUA & Finlândia & Suécia \\
Idade (horas) & 24.979 & 8.023 & 993,0 & 16.432 \\
\hline
\end{tabular}

Quadro 2 - Características técnicas dos modelos de "skidders" Table 2 - Characteristics of the skidders

\begin{tabular}{lcc}
\hline Características & Caterpillar 545 & Tigercat 630B \\
\hline Potência kW - (cv) & $168-(225)$ & $180-(240)$ \\
Especificação & 3306 DITA & Cummins \\
do motor & Caterpillar & 6CTA 8.3 \\
Massa (kg) & 15445 & 16000 \\
Originário & EUA & Canadá \\
Idade (horas) & 9.565 & 9.754 \\
\hline
\end{tabular}

\subsection{Determinação das dimensões do projeto interno}

Para a determinação ergonômica do projeto interno da cabine, as medidas foram coletadas com o auxílio de um equipamento de medida semelhante ao desenvolvido por Zander (1972), em que se mensuraram as distâncias do SIP até os órgãos de comandos e instrumentos nas três dimensões ( $x, y$ e $z$ ) e, posteriormente, representadas em um gráfico contendo as áreas de máximo e ótimo acesso aos comandos, esquematizados de acordo com as características antropométricas de um grupo de operadores brasileiros (FONTANA e SEIXAS, 2006) (Figura 1). O equipamento é composto por uma base de ferro maciça, que recebe uma das duas hastes reguláveis ligadas por uma junção. Ambas as hastes são graduadas em centímetros.

Para essa avaliação ergonômica foram coletados os dados com o assento em três posições diferenciadas, classificadas como limites: "próximo", "médio" e "extremo", adotando-se como referência o painel das máquinas em estudo. Posteriormente foi realizada a avaliação espacial da distribuição dos comandos para as três posições do assento, atribuindo-lhe conceitos (Quadro 3).

Além da avaliação ergonômica, aplicou-se individualmente um questionário para conhecer a opinião dos operadores sobre o acesso às máquinas, o regime de trabalho e a facilidade de execução dos comandos, dentre outros fatores. Nesse questionário, os operadores atribuíram valores de 1 a 10 , em ordem decrescente de avaliação, em que o maior valor indicava uma avaliação ótima e o menor, um pior resultado. O questionário foi aplicado a 25 operadores de "forwarders", com idade média de 31,5 anos e com 3,7 anos de experiência, e a 11 operadores de "skidders", com idade média de 38,4 anos e com 8,7 anos de experiência. Com os resultados obtidos foram calculadas as médias dos valores atribuídos pelos operadores a cada item e máquina florestal em estudo. O objetivo desse questionário foi confrontar as regiões de ótimo acesso determinadas na avaliação ergonômica com a opinião dos operadores.

\subsection{Determinação de medidas antropométricas}

Foram determinadas 20 medidas corporais de 34 operadores, sendo 23 operadores de "forwarders" e 11 de "skidders". Para a obtenção dessas medidas, utilizou-se uma cadeira portátil especial, de acordo com o apresentado por Serrano (1996), cuja precisão é da ordem de centímetros.

Para determinar as variações antropométricas dos operadores, a análise estatística constou do cálculo dos percentis, nos níveis de 5,0, 50,0 e 95,0\%. Também foram determinados a média, o desvio-padrão, o coeficiente de variação e o intervalo da medida no qual se encontram 90,0\% dos indivíduos. O coeficiente de variação e o intervalo da medida foram utilizados para efeito de comparação com dados antropométricos referentes aos países de origem dos equipamentos florestais (FONTANA e SEIXAS, 2006). Finalmente, esses valores possibilitaram a determinação gráfica das áreas de máximo e ótimo acesso (Figura 1).

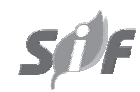

R. Árvore, Viçosa-MG, v.31, n.1, p.71-81, 2007 
Quadro 3 - Conceitos da avaliação espacial dos comandos nas coordenadas x-y e x-z

Table 3 - Concepts of command spatial evaluation in the coordinates $x-y$ and $x-z$

\begin{tabular}{cl}
\hline Conceito & \multicolumn{1}{c}{ Descrição } \\
\hline Ótimo & Comando situado na região de ótimo acesso nos dois planos \\
Muito bom & Comando situado na região de ótimo em um plano e de máximo no outro plano \\
Bom & Comando situado na região de máximo nos dois planos \\
Regular & Comando situado na região de máximo em um plano e fora no outro plano \\
Ruim & Comando situado em região externa aos dois planos \\
\hline
\end{tabular}

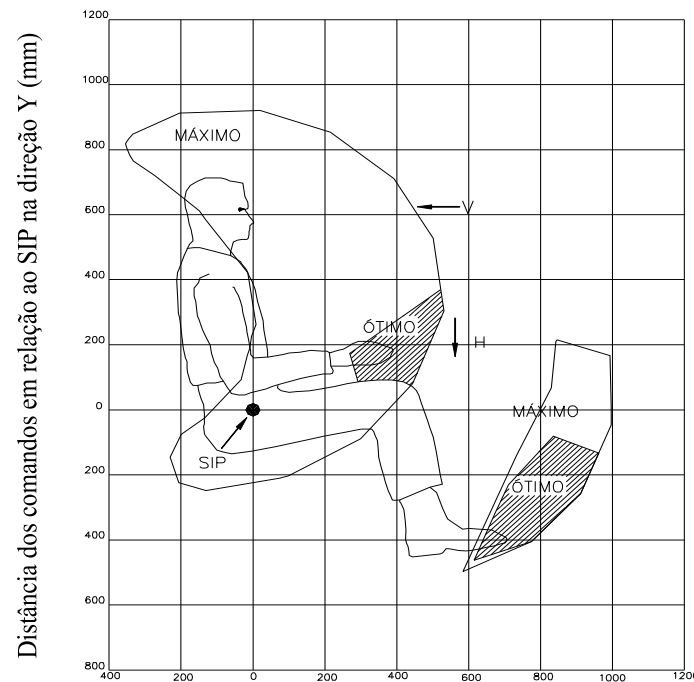

Distância dos comandos em relação ao SIP na direção X (mm)

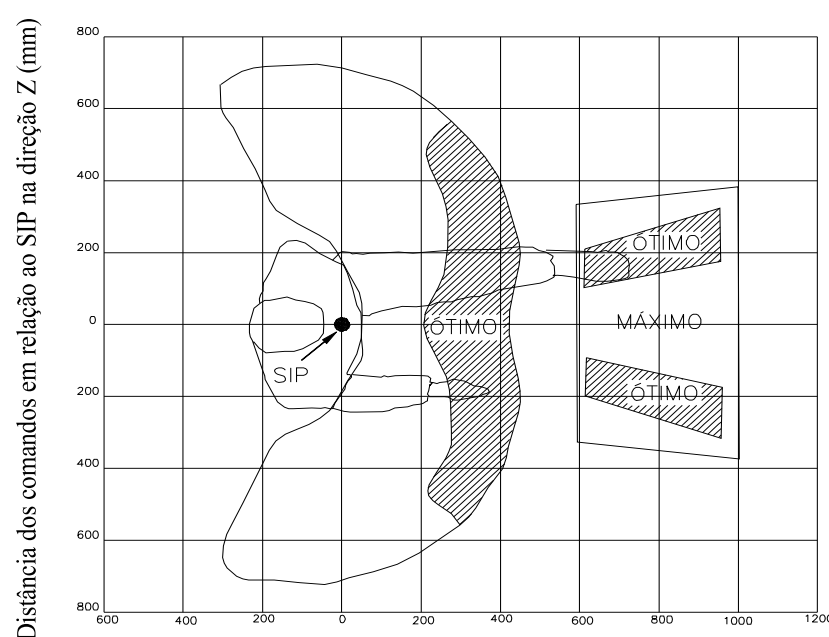

Distância dos comandos em relação ao SIP na direção X (mm)

Figura 1 - Áreas de máximo e ótimo acesso aos órgãos de comando, nas três dimensões.

Figure 1 -Areas of maximum and excellent access to command instruments, in three dimensions.

\section{RESULTADOS E DISCUSSÃO}

\subsection{Antropometria}

Os resultados antropométricos obtidos na amostragem de um conjunto de 34 operadores de máquinas florestais são apresentados no Quadro 4.

Segundo Fontana e Seixas (2006), quando comparados os dados antropométricos dos operadores de máquinas florestais das empresas em estudo com os operadores do sul dos Estados Unidos, os resultados apontaram que há diferenças com o biótipo geral dos operadores da região abrangida por esta pesquisa.

\subsection{Ergonomia}

\subsection{1. "Forwarder" Timberjack modelo 1210B}

Analisando a distribuição espacial do posicionamento dos comandos com o assento nas três regiões avaliadas, observou-se que os principais comandos utilizados durante a jornada de trabalho, indiferentemente do posicionamento do assento, receberam o conceito “ótimo" (Quadro 5), fato explicado por estarem fixos ao assento da máquina (Figura 2).

Entre os comandos que receberam o conceito classificado como "ótimo", encontram-se os "joysticks" do comando da grua dos lados esquerdo e direito, volante (joystick) e o interruptor de direção da máquina. Os pedais de acelerador e o freio receberam o conceito "ruim" nas três situações avaliadas, exigindo um esforço adicional para que o operador consiga manipulá-los. Entretanto, observou-se que, para os diferentes posicionamentos do assento, existia uma diferença na distribuição dos comandos quando considerada a somatória dos conceitos "ótimo", "muito bom" e "bom", sendo a maior porcentagem obtida com o posicionamento em extremo (60,0\%), fato esse explicado pela distribuição dos comandos do lado direito do operador. 
Quadro 4 - Padrão antropométrico de um conjunto de operadores de máquinas florestais Table 4 - Anthropometric pattern of a group of forest machine operators

\begin{tabular}{|c|c|c|c|c|c|c|}
\hline \multirow[t]{2}{*}{ Medidas } & \multirow[t]{2}{*}{ Média $(\mathrm{cm})$} & \multirow[t]{2}{*}{ Desvio-padrão (cm) } & \multirow[t]{2}{*}{$\mathrm{CV}(\%)$} & \multicolumn{3}{|c|}{ Percentil } \\
\hline & & & & $5 \%$ & $50 \%$ & $95 \%$ \\
\hline Altura em pé & 170,7 & 6,0 & 4,0 & 159,9 & 168,0 & 181,4 \\
\hline Altura olho-chão & 160,2 & 5,0 & 3,0 & 149,9 & 157,0 & 170,4 \\
\hline Altura ombro-chão & 144,1 & 5,0 & 3,0 & 135,8 & 141,5 & 152,3 \\
\hline Altura cotovelho-chão & 106,5 & 4,0 & 4,0 & 101,9 & 106,0 & 111,1 \\
\hline Assento-pé & 45,7 & 3,0 & 7,0 & 44,4 & 45,5 & 47,0 \\
\hline Sacro-poplítea & 48,4 & 4,0 & 8,0 & 46,4 & 47,5 & 50,3 \\
\hline Assento-cabeça & 84,7 & 4,0 & 5,0 & 81,1 & 84,0 & 88,2 \\
\hline Assento-olho & 73,9 & 5,0 & 7,0 & 70,2 & 73,0 & 77,5 \\
\hline Assento-ombro & 61,5 & 4,0 & 7,0 & 58,8 & 61,0 & 64,2 \\
\hline Assento-cotovelo & 20,7 & 2,0 & 10,0 & 20,0 & 20,1 & 21,3 \\
\hline Assento-altura da coxa & 15,2 & 2,0 & 13,0 & 14,9 & 15,0 & 15,5 \\
\hline Poplítea-extremidade do joelho & 11,9 & 2,0 & 17,0 & 11,5 & 11,7 & 12,1 \\
\hline Comprimento do pé & 25,4 & 1,0 & 4,0 & 25,0 & 25,1 & 25,8 \\
\hline Largura do pé & 9,8 & 1,0 & 10,0 & 9,5 & 9,7 & 9,9 \\
\hline Largura dos ombros & 47,3 & 3,0 & 6,0 & 45,7 & 46,5 & 48,9 \\
\hline Cotovelo-extremidade dedo indicador & 47,4 & 2,0 & 4,0 & 46,4 & 46,5 & 48,3 \\
\hline Cotovelo-extremidade mão fechada & 39,4 & 2,0 & 5,0 & 38,6 & 39,0 & 40,2 \\
\hline Comprimento do braço & 85,2 & 5,0 & 6,0 & 80,8 & 83,5 & 89,7 \\
\hline Massa (kg) & 85,2 & 8,0 & 9,0 & 77,0 & 83,0 & 93,5 \\
\hline
\end{tabular}

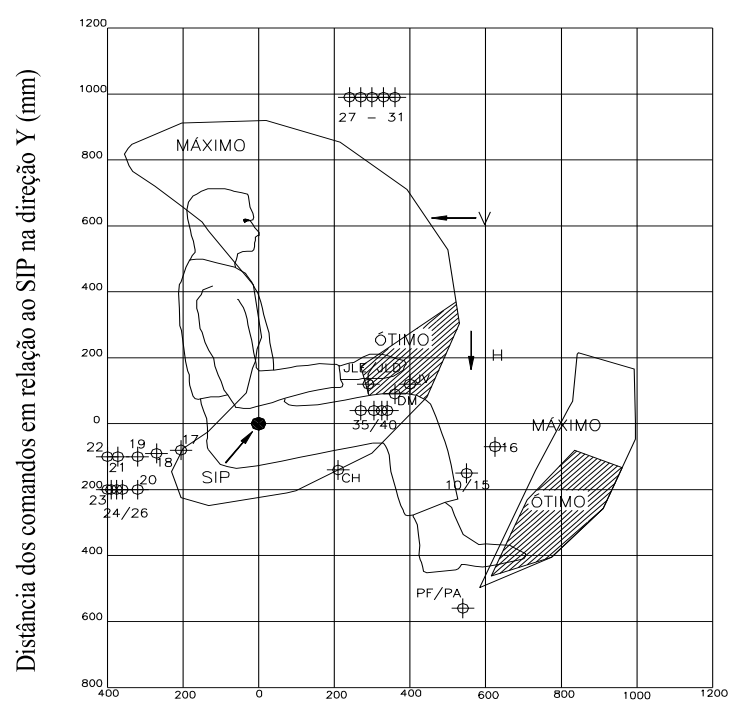

Distância dos comandos em relação ao SIP na direção X $(\mathrm{mm})$

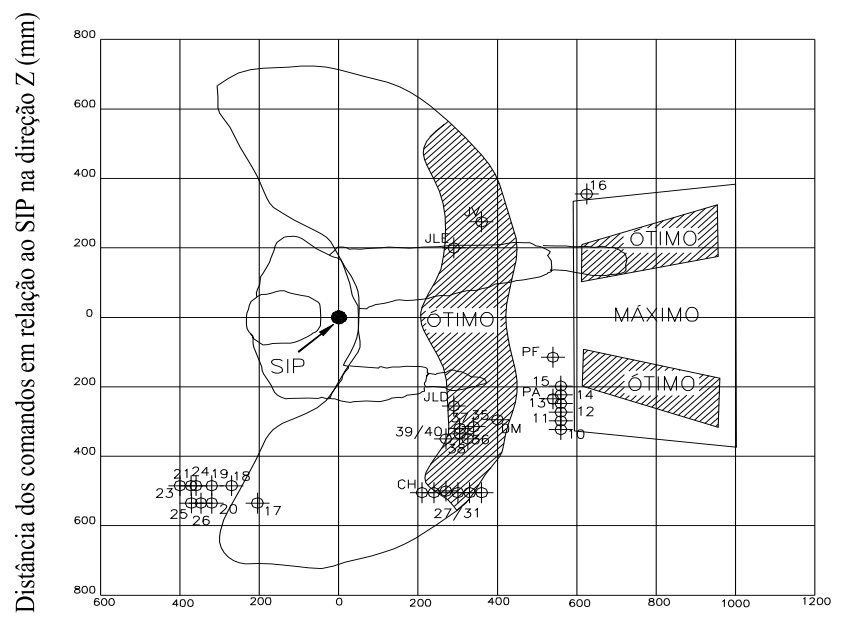

Distância dos comandos em relação ao SIP na direção X ( $\mathrm{mm})$

CH - Interruptor de Ignição (Chave); 2 - Luz avisadora de Sinal de deslocamento; 3 - Luz dos Faróis de trabalho; 4 - Luz dos Faróis Alto; 5 - Luz do Sistema Elétrico (Alternador); 6 - Luz de Pressão do Óleo de motor; 7 - Luz do Freio de Estacionamento; 8 - Luz de Pressão de Abastecimento Óleo; 9 - Luz do Nível de Combustível; 10 - Interruptor dos Faróis; 11 - Interruptor do Controle médio/ máximo; 12 - Buzina; 13 - Interruptor de Piscas Intermitentes; 14 - Inter. Lavadores dos Vidros; 15 - Interruptor do Pisca; 16 - Inter. Degrau de Acesso a Cabine; 17 - Interruptor de Parada de Emergência; 18 - Interruptor do Controle de Temperatura; 19 - Inter. Trava do Freio de Estacionamento; 20 - Interruptor do Condicionador de Ar; 21 - Interruptor do bloqueio 6x6; 22 - Interruptor de RPM; 23 - Interruptor de Trava Chassis; 24 - Interruptores do Movimento Malhal; 25 - Inter. Limpador de Pára-brisa Traseiro Esq; 26 - Inter. Limpador de Pára-brisa Traseiro Intermitente; 27 - Interruptor de Faróis de Trabalho; 28, 29, 30 e 31 - Interruptor de Faróis de Trabalho; JV - "Joystick" do Volante da Máquina; JLE - "Joystick" do Comando da Grua; JLD - "Joystick" do Comando da Grua; 35 - Interruptor de Sentido de Deslocamento; 36 - Interruptor do Limpador de Pára-brisa; PF - Pedal de Freio; PA - Pedal de Acelerador

Figura 2 - Localização dos órgãos de comandos do "forwarder" Timberjack 1210B, nas três dimensões.

Figure 2-Command position of Timberjack 1210B, in three dimensions. 
Quadro 5-Distribuição espacial dos 35 comandos do "forwarder" Timberjack $1210 \mathrm{~B}$ na avaliação espacial das coordenadas $\mathrm{x}-\mathrm{y}$ e $\mathrm{x}-\mathrm{z}$

Table 5 - Spatial distribution of the thirty-five commands of Timberjack $1210 B$ in the spatial evaluation of coordinates $x-y$ and $x-z$

\begin{tabular}{lccc}
\hline Conceito & \multicolumn{3}{c}{ Posicionamento do assento } \\
\cline { 2 - 4 } & Próximo & Médio & Extremo \\
\hline Ótimo & $4(11,5 \%)$ & $4(11,5 \%)$ & $4(11,5 \%)$ \\
Muito bom & $6(17,0 \%)$ & $6(17,0 \%)$ & - \\
Bom & $6(17,0 \%)$ & $7(20,0 \%)$ & $17(48,5 \%)$ \\
Regular & $6(17,0 \%)$ & & $5(14,3 \%)$ \\
Ruim & $13(37,5 \%)$ & $18(51,5 \%)$ & $9(25,7 \%)$ \\
\hline Total & $35(100,0 \%)$ & $35(100,0 \%)$ & $35(100,0 \%)$ \\
\hline
\end{tabular}

\subsection{2. "Forwarder" Timberjack modelo 1710D}

Com relação à distribuição espacial dos 51 comandos nas três posições do assento (Figura 3), verificouse que apenas dois $(4,0 \%)$ desses comandos receberam o conceito "ótimo" ("joysticks" do comando da grua dos lados esquerdo e direito), um só comando foi sempre

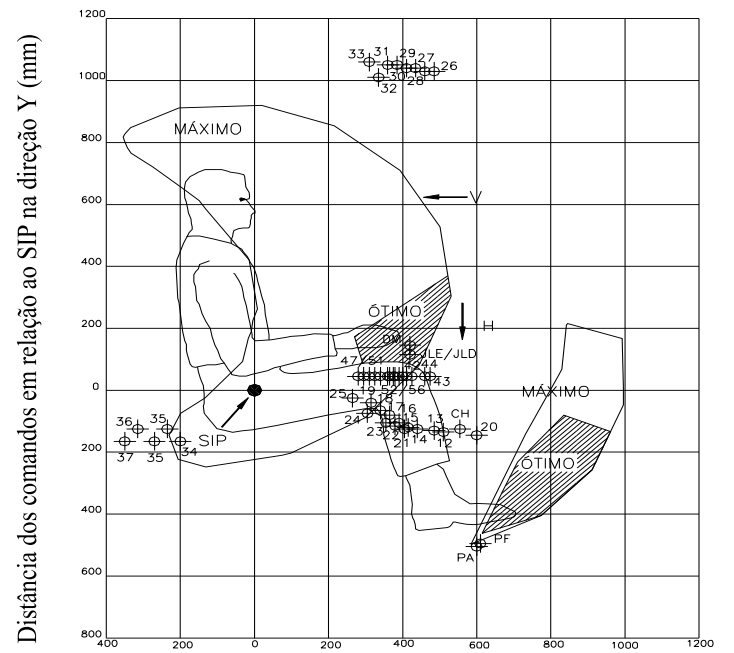

Distância dos comandos em relação ao SIP na direção X (mm) classificado como "bom" (interruptor de direção da máquina) e dois foram conceituados como "regulares" (os pedais do acelerador e do freio), sendo que poderiam vir a ocasionar um desconforto para o operador durante a operação (Quadro 6). Comparando a distribuição espacial dos comandos conforme o posicionamento do assento, observou-se que, com o posicionamento do assento em "próximo", apenas 56,9\% deles conseguiram conceitos entre "bom" e "ótimo"; em posição "médio," foram 43,0\% e, em "extremo", 49,0\% do total dos comandos.

\subsection{3. "Forwarder" Valmet modelo 890.2}

Analisando o "forwarder" 890.2 em relação ao posicionamento espacial dos comandos nas três posições do assento, verificou-se que, dos 48 comandos, apenas dois $(4,0 \%)$ foram classificados como "ótimo" (Quadro 7). Independentemente do posicionamento do assento, observou-se que os pedais de freio e o acelerador foram classificados, respectivamente, como "muito bom" e "regular" (Figura 4).

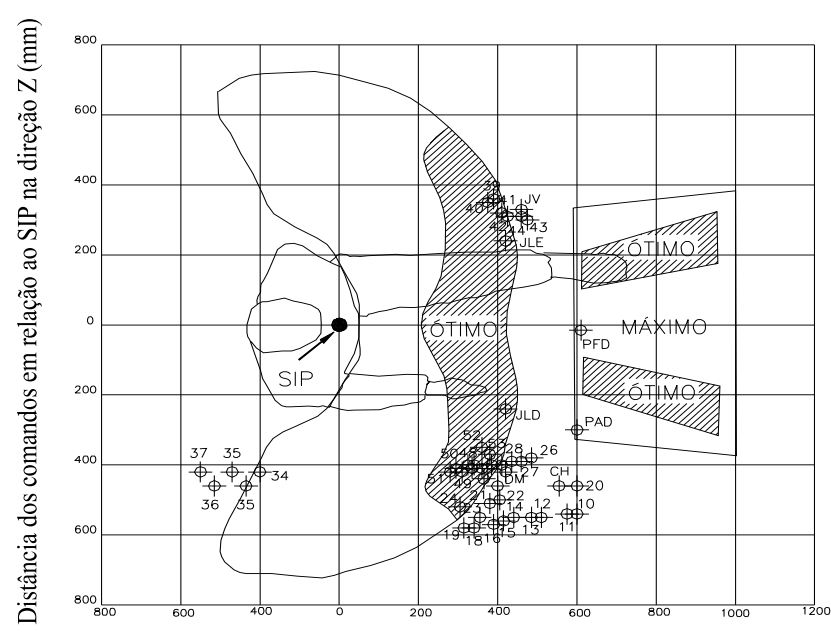

Distância dos comandos em relação ao SIP na direção X (mm)

CH - Interruptor de Ignição (Chave); 2 - Luz avisadora de Sinal de deslocamento; 3 - Luz dos Faróis de trabalho; 4 - Luz dos Faróis Alto; 5 - Luz do Sistema Elétrico (Alternador); 6 - Luz de Pressão do Óleo de motor; 7 - Luz do Freio de Estacionamento; 8 - Luz de Pressão do Acumulador; 9 - Luz de Pressão Óleo Transmissão; 10 - Interruptor do Pisca; 11 - Buzina; 12 - Inter. Degrau de Acesso a Cabine; 13 - Interruptor do bloqueio 6x6; 14 - Inter. Lavador Pará-brisa; 15, 16, 17, 18 e 19 - Inter. Limpador de Pára-brisa; 20 - Acendedor de Cigarro; 21 - Interruptor de Luz Alta; 22 - Interruptor dos Faróis; 23 - Interruptor da Direção Máquina; 24 - Inter. Freio de Estacionamento; 25 - Inter. Parada de Emergência; 26, 27, 28, 29, 30, 31 e 32 - Interruptor de Faróis de Serviço; 33 - Inter. do Ventilador de Teto; 34 - Inter. Posicionamento Ar; 35 - Inter. Controle Velocidade Temp.; 35- Inter. Controle Temp. (quente/frio); 36 - Interruptor de Saídas; 37 - Inter. Desembaçador Interno Cabine; DM - Monitor ("Display"); JV - "Joystick" de Direção (Volante); 39 - Botão de Levantar Malhal; 40 - Botão de Baixar Malhal; 41 - Botão Desacoplar Freio Torcional; 42 - Botão de Rotação de Trabalho; 43 - Botão Bloqueio acionar Dif.(6x6); 44 -Botão Bloqueio Desacionar Dif. (6x6); JLE - "Joystick" do Comando da Grua; JLD - "Joystick" do Comando da Grua; 47 - Botão de Acesso Display (A); 48 - Botão de Acesso Display (B); 49 - Botão de Acesso Display (C); 50 - Botão de Acesso Display (D); 51 - Botão de Acesso Display (E); 52 - Botão de Sentido de Deslocamento; 53 - Botão de Velocidades Altas/Baixas; 54 - Botão da Grua ligada/desligada; 55 - Botão de Aquecimento Acento; 56 - Botão Limp. Pára-brisa de Carga; PF - Pedal de Freio; PA - Pedal de Acelerador Dianteiro.

Figura 3 - Localização dos órgãos de comandos do "forwarder" Timberjack 1710D, nas três dimensões.

Figure 3 - Command position of Timberjack 1710D, in three dimensions.

R. Árvore, Viçosa-MG, v.31, n.1, p.71-81, 2007 
Quadro 6-Distribuição espacial dos 51 comandos do "forwarder" Timberjack 1710D na avaliação espacial das coordenadas $\mathrm{x}-\mathrm{y}$ e $\mathrm{x}-\mathrm{z}$

Table 6-Spatial distribution of fifty and commands of Timberjack $1710 D$ in the spatial evaluation of coordinates $x$ $y$ and $x-z$

\begin{tabular}{lccr}
\hline Conceito & \multicolumn{2}{c}{ Posicionamento do assento } \\
\cline { 2 - 4 } & Próximo & Médio & Extremo \\
\hline Ótimo & $2(4,0 \%)$ & $2(4,0 \%)$ & $2(4,0 \%)$ \\
Muito bom & $13(25,5 \%)$ & $11(21,5 \%)$ & $11(21,5 \%)$ \\
Bom & $14(27,4 \%)$ & $9(17,5 \%)$ & $12(23,5 \%)$ \\
Regular & $8(15,7 \%)$ & $5(10,0 \%)$ & $4(8,0 \%)$ \\
Ruim & $14(27,4 \%)$ & $24(47,0 \%)$ & $22(43,0 \%)$ \\
\hline Total & $51(100,0 \%)$ & $51(100,0 \%)$ & $51(100,0 \%)$ \\
\hline
\end{tabular}

Quadro 7-Distribuição espacial dos 48 comandos do "forwarder" Valmet 890.2 na avaliação espacial das coordenadas $\mathrm{x}-\mathrm{y}$ e $\mathrm{x}-\mathrm{z}$

Table 7 - Spatial distribution of the forty-eight commands of Valmet 890.2 in the spatial evaluation of coordinates $x-y$ and $x-z$

\begin{tabular}{|c|c|c|c|}
\hline \multirow[t]{2}{*}{ Conceito } & \multicolumn{3}{|c|}{ Posicionamento do assento } \\
\hline & Próximo & Médio & Extremo \\
\hline Ótimo & $2(4,0 \%)$ & $2(4,0 \%)$ & $2(4,0 \%)$ \\
\hline Muito Bom & $19(39,5 \%)$ & $21(43,7 \%)$ & $19(39,5 \%)$ \\
\hline Bom & $9(19,0 \%)$ & $9(19,0 \%)$ & $4(8,5 \%)$ \\
\hline Regular & $7(14,5 \%)$ & $12(25,0 \%)$ & $15(31,5 \%)$ \\
\hline Ruim & $11(23,0 \%)$ & $4(8,3 \%)$ & $8(16,0 \%)$ \\
\hline Total & $48(100,0 \%)$ & $48(100,0 \%)$ & $48(100,0 \%)$ \\
\hline
\end{tabular}

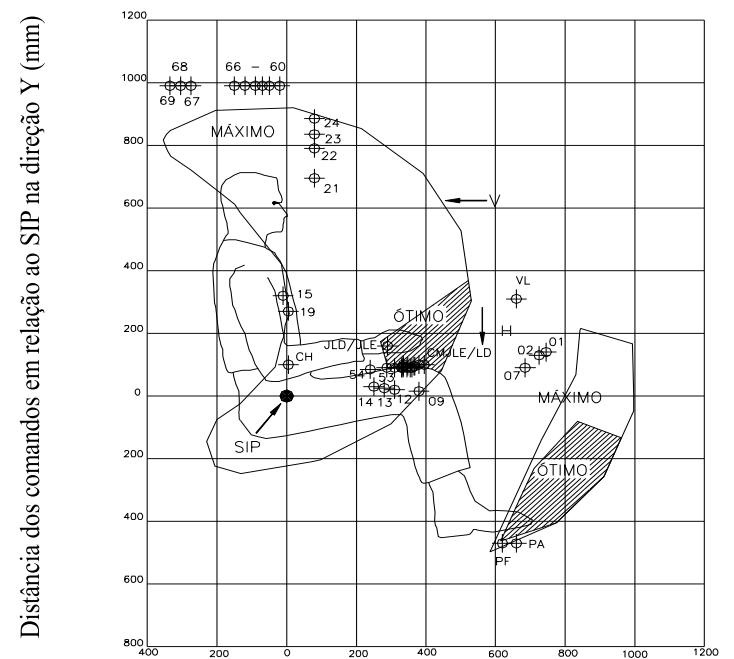

Distância dos comandos em relação ao SIP na direção X (mm)

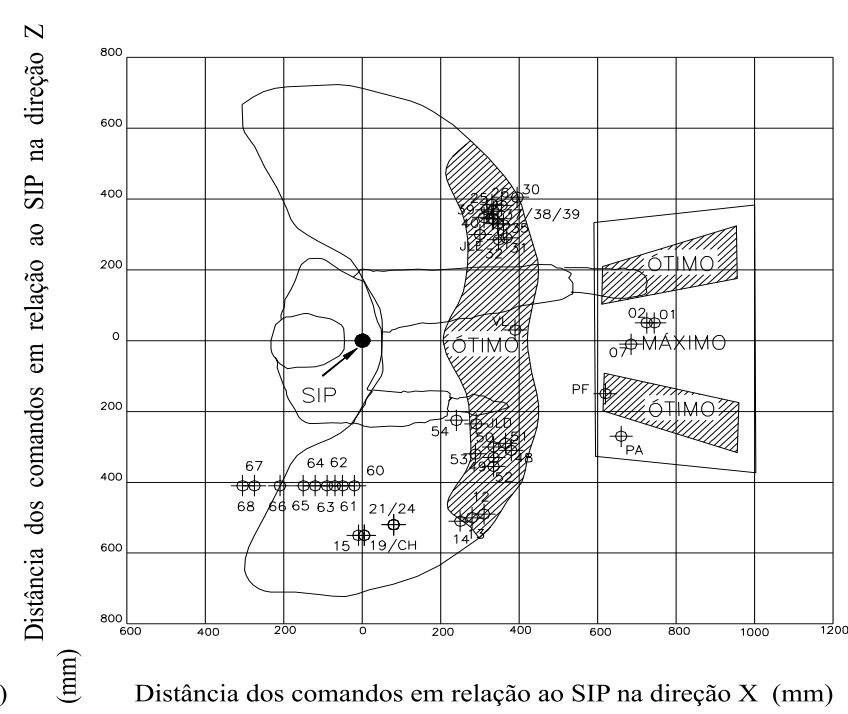

1 - Inter. Sentido de Deslocamento; 2 - Inter. Farol (Alto/Baixo); 3 - Luz dos Faróis de Trabalho; 4 - Luz do Controle de EHC; 5 - Luz do Sentido de Deslocamento; 6 - Luz de Farol Alto; 7 - Relógio do Horímetro; 9 - Inter. Emergência; 12 - Inter. Limpador do Para-brisa Frontal; 13 - Inter. Buzina; 14 - Inter. Luz de Trabalho; 15 - Interruptor de Parada de Emergência; 19 - Inter. Luz do Freio de Estacionamento; CH - Inter. de Ignição (Chave); 21 - Inter. Velocidade do Ventilador; 22 - Inter. Liga/Desliga; 23 - Inter. Controle de Temperatura; 24 - Inter. Posicionamento Circulação AR; 25 - Botão Inch - painel de controle do joystick; 26 - Botão de Neutro; 29- Botão da Mudança Janela Programa; 30 - Botão de Rotações de Trabalho; 31 - Botão Trava do Diferencial Dianteira; 32 - Botão Trava do Diferencial Traseira; 35 - Botão de Deslocamento Frete; 36 - Botão de Deslocamento Trás; 37 - Botão de ajuda; 38 - Botão de Enter; 39 - Botão de Shift; 40 - Botão de ESC; JDE - "Joystick" do Comando da Grua (LE); DM- Monitor Display; 48 - Botão Limp. Pára-brisa Intermitente; 49 - Botão de Operação da Grua; 50 - Botões de Setas; 51 - Botão do Menu; 52 - Botão do Menu A - D; 53 - Botão de Transporte; 54 - Botão do Giro do Assento; JLD - "Joystick" do Comando da Grua; PF - Pedal de Freio; PA - Pedal de Acelerador; 60 - Inter. Luz Alta; 61 - Inter. Luzes Serviço do Teto Frontal; 62 Inter. Luzes Lateral Direita Cabine; 63 - Inter. Luzes Lateral Esquerda Cabine.

Figura 4 - Localização dos órgãos de comandos do "forwarder" Valmet 890.2, nas três dimensões.

Figure 4-Command position of Valmet 890.2, in three dimensions.

A somatória da porcentagem do número de comandos com conceitos "ótimo", "muito bom"e "bom" resultou em $62,5 \%$, com o posicionamento do assento em "próximo", 66,7\% em "médio" e 52,0\% em "extremo". No caso desse modelo, observa-se que a melhor classificação foi obtida com o posicionamento em "médio", o que permite a sua melhor adequação para um número maior de operadores, haja vista que a análise feita neste trabalho se baseou no biótipo do operador médio. Em relação aos comandos que foram classificados como "regular" e "ruim", verificou-se que, na maioria das vezes, eram utilizados, principalmente em jornada de trabalho noturna, exemplo dos interruptores de farol. 


\subsection{4. "Forwarder" Volvo modelo A25C}

Em relação à distribuição espacial dos 31 comandos nas três posições do assento (Quadro 8), observouse que apenas $6,5 \%$ dos comandos receberam conceito "ótimo" e 13,0\%, "muito bom" (Figura 5). O volante e o pedal de freio destacaram-se entre os comandos que receberam o conceito "ótimo", e os "joysticks" do comando da grua dos lados esquerdo e direito e o pedal do acelerador classificaram-se como "muito bom" nas três posições do assento.

Quando comparada a distribuição dos comandos nos diferentes posicionamentos do assento com a dos demais modelos, verificou-se que esse "forwarder" apresentou a menor porcentagem dos comandos em posição adequada.

\subsection{5. "Skidder" Caterpillar modelo 545}

O "skidder" Cat 545 dos 24 comandos avaliados, apenas um recebeu o conceito "ótimo" ("joystick" do comando da grua) (Quadro 9), nos três posicionamentos do assento (Figura 6). Observando-se a distribuição dos comandos, constatando-se que 70,0\% dos comandos receberam conceitos "ótimo", "muito bom" ou "bom" para o assento posicionado em "extremo", 54,5\% em "médio" e 46,0\% em "próximo". Dois comandos utilizados durante a jornada de trabalho encontravam-se localizados em uma região com conceito "ruim": os pedais de freio e acelerador.

Quadro 8-Distribuição espacial dos 31 comandos do "forwarder" Volvo A25C na avaliação espacial das coordenadas $\mathrm{x}-\mathrm{y}$ e $\mathrm{x}-\mathrm{z}$

Table 8 - Spatial distribution of the thirty-one commands of Volvo A25C in the spatial evaluation of coordinates $x-y$ and $x-z$

\begin{tabular}{lccc}
\hline Conceito & \multicolumn{3}{c}{ Posicionamento do assento } \\
\cline { 2 - 4 } & Próximo & Médio & Extremo \\
\hline Ótimo & $2(6,5 \%)$ & $2(6,5 \%)$ & $2(6,5 \%)$ \\
Muito bom & $4(13,0 \%)$ & $4(13,0 \%)$ & $4(13,0 \%)$ \\
Bom & $5(16,0 \%)$ & $7(22,5 \%)$ & $6(19,0 \%)$ \\
Regular & $11(35,5 \%)$ & $7(22,5 \%)$ & $2(6,5 \%)$ \\
Ruim & $9(29,0 \%)$ & $11(35,5 \%)$ & $17(55,0 \%)$ \\
\hline Total & $31(100,0 \%)$ & $31(100,0 \%)$ & $31(100,0 \%)$ \\
\hline
\end{tabular}

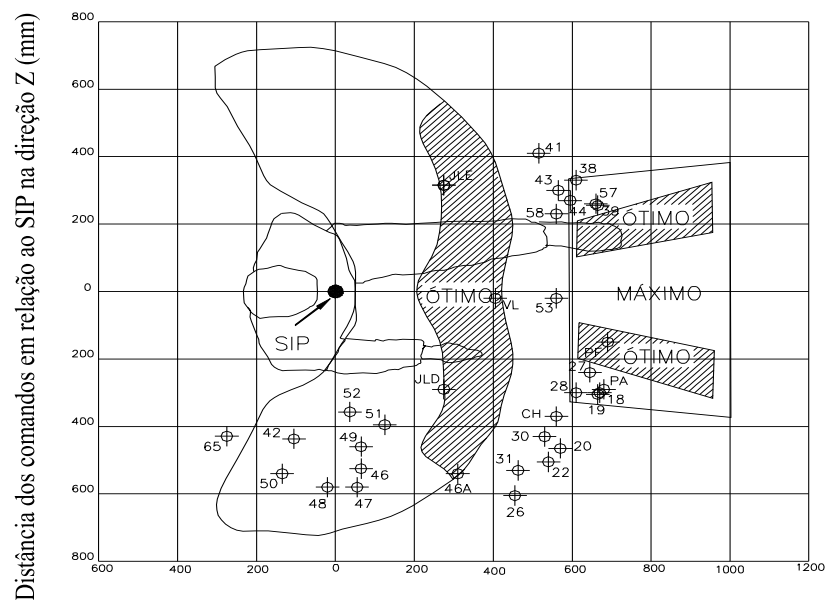

Distância dos comandos em relação ao SIP na direção X (mm)

Distância dos comandos em relação ao SIP na direção X $(\mathrm{mm})$

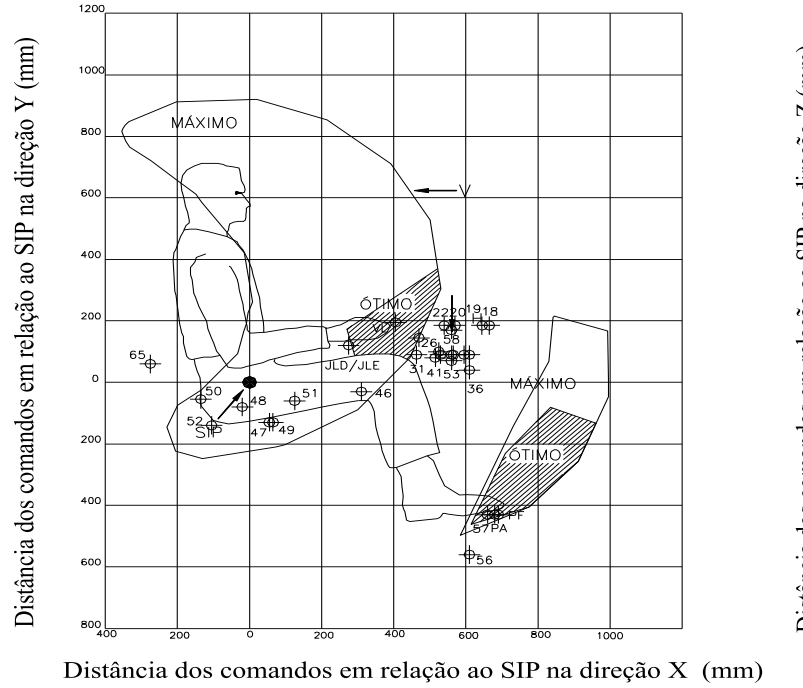

CH - Inter. Ignição (Chave); 1 - Inter. Sentido de Deslocamento; 2 - Inter. Farol (Alto/Baixo); 3 - Luz dos Faróis de Trabalho; 4 - Luz do Controle de EHC; 5 - Luz do Sentido de Deslocamento; 6 - Luz de Farol Alto; 7 - Relógio do Horímetro; 9 - Inter. Emergência; 12 - Inter. Limpador do Para-brisa Frontal; 13 - Inter. Buzina; 14 - Inter. Luz de Trabalho; 15 - Interruptor de Parada de Emergência; 19 - Inter. Luz do Freio de Estacionamento; 21 - Inter. Velocidade do Ventilador; 22 - Inter. Liga/Desliga; 23 - Inter. Controle de Temperatura; 24 - Inter. Posicionamento Circulação AR; 25 - Botão Inch - painel de controle do joystick; 26 - Botão de Neutro; 29- Botão da Mudança Janela Programa; 30 - Botão de Rotações de Trabalho; 31 - Botão Trava do Diferencial Dianteira; 32 - Botão Trava do Diferencial Traseira; 35 - Botão de Deslocamento Frete; 36 - Botão de Deslocamento Trás; 37 - Botão de ajuda; 38 - Botão de Enter; 39 - Botão de Shift; 40 - Botão de ESC; JDE - "Joystick" do Comando da Grua (LE); DM- Monitor Display; 48 - Botão Limp. Pára-brisa Intermitente; 49 - Botão de Operação da Grua; 50 - Botões de Setas; 51 - Botão do Menu; 52 - Botão do Menu A - D; 53 - Botão de Transporte; 54 - Botão do Giro do Assento; JLD - "Joystick" do Comando da Grua; PF - Pedal de Freio; PA - Pedal de Acelerador; 60 - Inter. Luz Alta; 61 - Inter. Luzes Serviço do Teto Frontal; 62 Inter. Luzes Lateral Direita Cabine; 63 - Inter. Luzes Lateral Esquerda Cabine.

Figura 5 - Localização dos órgãos de comandos do "forwarder" Volvo A25C, nas três dimensões.

Figure 5 - Command position of Volvo A25C, in three dimensions. 
Quadro 9-Distribuição espacial dos 24 comandos do "skidder" Caterpillar 545 na avaliação espacial das coordenadas $\mathrm{x}-\mathrm{y}$ e $\mathrm{x}-\mathrm{z}$

Table 9 -Spatial distribution of the twenty-four commands of Caterpillar 545 in the spatial evaluation of coordinates $x-y$ and $x-z$

\begin{tabular}{lccc}
\hline Conceito & \multicolumn{2}{c}{ Posicionamento do assento } \\
\cline { 2 - 4 } & Próximo & Médio & Extremo \\
\hline Ótimo & $1(4,0 \%)$ & $3(12,5 \%)$ & $1(4,0 \%)$ \\
Muito bom & $5(21,0 \%)$ & $5(21,0 \%)$ & $1(4,0 \%)$ \\
Bom & $5(21,0 \%)$ & $5(21,0 \%)$ & $16(67,0 \%)$ \\
Regular & $3(12,5 \%)$ & $3(12,5 \%)$ & $2(8,5 \%)$ \\
Ruim & $10(41,5 \%)$ & $8(33,0 \%)$ & $4(16,5 \%)$ \\
\hline Total & $24(100,0 \%)$ & $24(100,0 \%)$ & $24(100,0 \%)$ \\
\hline
\end{tabular}

Apesar da quantidade de comandos que receberam o conceito classificado como "regular" e "ruim", há que se considerar que boa parte deles era acionada com menor freqüência, à exceção dos pedais de freio e do acelerador.

\subsection{6. "Skidder" Tigercat modelo 630B}

Quanto ao "skidder" Tigercat 630B, verificou-se que, com o assento posicionado em "próximo", 63,0\% dos comandos atingiram os conceitos entre "bom" e "ótimo" (Quadro 10). Os comandos utilizados durante toda a jornada de trabalho e classificados como "bom", nas três posições do assento avaliadas, foram: pedal de acelerador para frente, "joystick" para controle da garra, alavanca de controle da lâmina, alavanca de câmbio e o volante. O único comando encontrado em posição "regular" foi o pedal do acelerador de ré (Figura 7). Quando comparada a distribuição espacial dos comandos nas três posições do assento, entre os "skidders" Catterpillar 545 e o Tigercat 630B, observou-se que o "skidder" Tigercat 630B somente apresentou melhor distribuição dos seus comandos com a localização do assento em "próximo" (63,0\% contra 46,0\%), com o modelo da Caterpillar sendo mais bem avaliado nas demais posições do assento.

\subsubsection{Análise comparativa entre os modelos de máquinas}

Para simplificar a comparação entre os tratores florestais, os resultados da localização dos comandos com o assento na posição "média" (Quadros 5, 6, 7, 8,9 e 10) foram agregados em dois únicos conceitos mais amplos: A ("ótimo" + "muito bom" + "bom") e B ("regular" + "ruim") (Quadro 11).
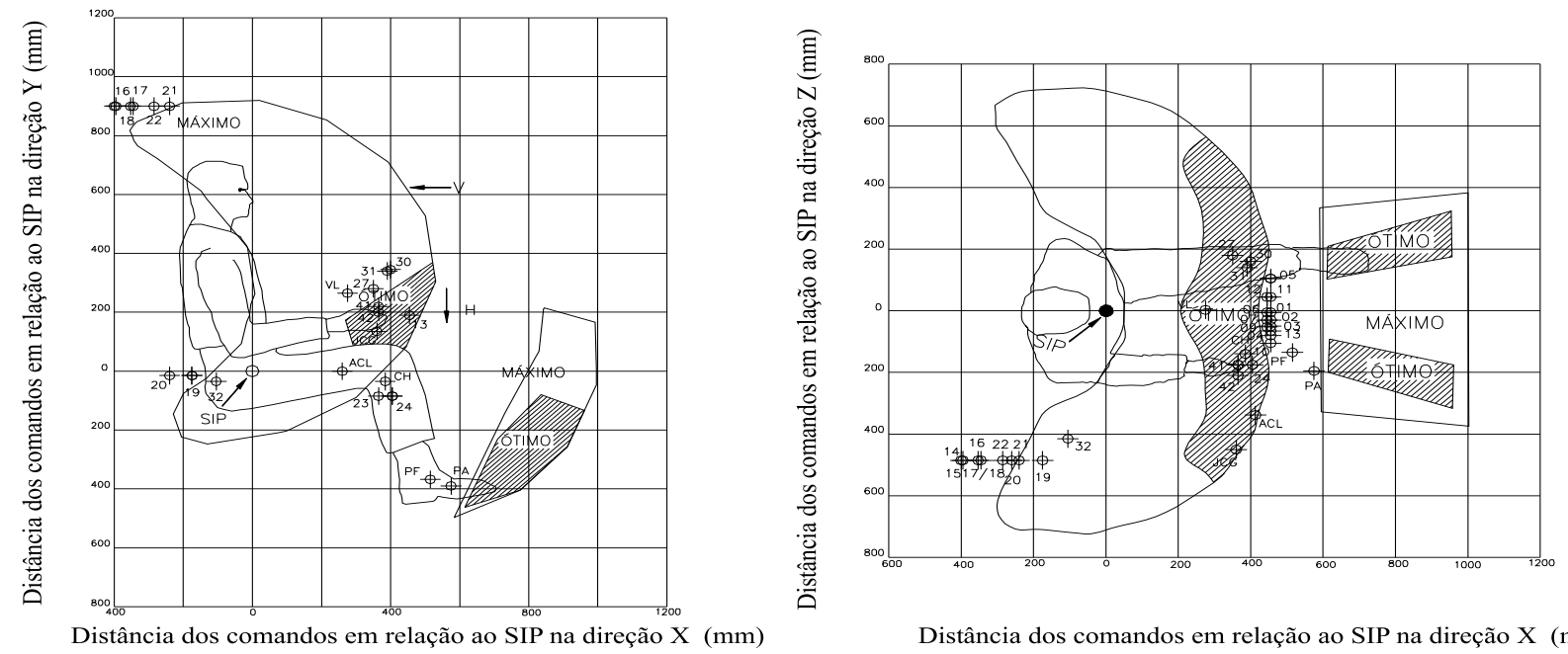

Distância dos comandos em relação ao SIP na direção X (mm)

1- Luz de Pressão do Óleo de motor: 2 - Luz do Freio de Estacionamento; 3 - Luz da Pressão Óleo de Freio; 4 - Luz do Sistema Elétrico; 5 - Luz do Nível de Combustível;6 - Luz dos Braços Automático da Garra; 7 - Luz do Filtro do Óleo de Transmissão; 8 - Luz da Trava do Diferencial; 9 - Relógio Temperatura Motor; 10 - Relógio Temperatura Óleo da Transmissão; 11 - Relógio Temperatura Óleo Hidráulico; 12 - Relógio Nível de Combustível; 13 - Inter. do Visor Digital/Horímetro/RPM/Marcha; 14 - Inter. Faróis Dianteiros; 15 - Inter. Faróis Traseiros; 16 - Inter. Lavadores dos Vidros; 17 - Inter. Limpador de Pará-brisa Traseiro; 18 - Inter. Lavador Pará-brisa Dianteiro; 19 - Inter. Aquecedor e Condicionador de Ar; 20 - Inter. Controle de Temperatura; 21 - Inter. Ventilador Traseiro; 22 - Inter. Ventilador Dianteiro; 23 - Alavanca do Freio de Estacionamento (Acionada); 27 - Cambio (Avante); 30 - Inter. Mudança Ascendente de Marcha; 31 - Inter. Mudança Redução de Marcha; 32 - Inter. Trava do Diferencial; 34 - Posicionamento do Volante; ACL - Alavanca de Controle Lâmina; CH - Inter. Ignição (Chave); JCG - "Joystick” do Controle da Garra; 41 - Inter. Auxiliar da Pinça; 42 - Inter. Auxiliar do Torque.

Figura 6 - Localização dos órgãos de comandos do "skidder” Caterpillar 545, nas três dimensões.

Figure 6-Command position of Caterpillar 545, in three dimensions. 
Quadro 10 - Distribuição espacial dos 19 comandos do "skidder" Tigercat 630B na avaliação espacial das coordenadas $\mathrm{x}-\mathrm{y}$ e $\mathrm{x}-\mathrm{z}$

Table 10 - Spatial distribution of the nineteen commands of Tigercat $630 B$ in the spatial evaluation of coordinates $x-y$ and $x-z$.

\begin{tabular}{|c|c|c|c|}
\hline \multirow[t]{2}{*}{ Conceito } & \multicolumn{3}{|c|}{ Posicionamento do assento } \\
\hline & Próximo & Médio & Extremo \\
\hline Ótimo & $4(21,0 \%)$ & & \\
\hline Muito bom & $2(10,5 \%)$ & & \\
\hline Bom & $6(31,5 \%)$ & $5(26,0 \%)$ & $5(26,0 \%)$ \\
\hline Regular & $4(21,0 \%)$ & $7(37,0 \%)$ & $7(37,0 \%)$ \\
\hline Ruim & $3(16,0 \%)$ & $7(37,0 \%)$ & $7(37,0 \%)$ \\
\hline Total & $19(100,0 \%)$ & $19(100,0 \%)$ & $19(100,0 \%)$ \\
\hline
\end{tabular}

A consideração de que o "forwarder” é uma máquina mais bem projetada ergonomicamente que o "skidder" não se confirmou, de acordo com os resultados dos modelos de tratores deste estudo. Com base nos dados do Quadro 11, o “forwarder" Valmet 890.2 apresentou

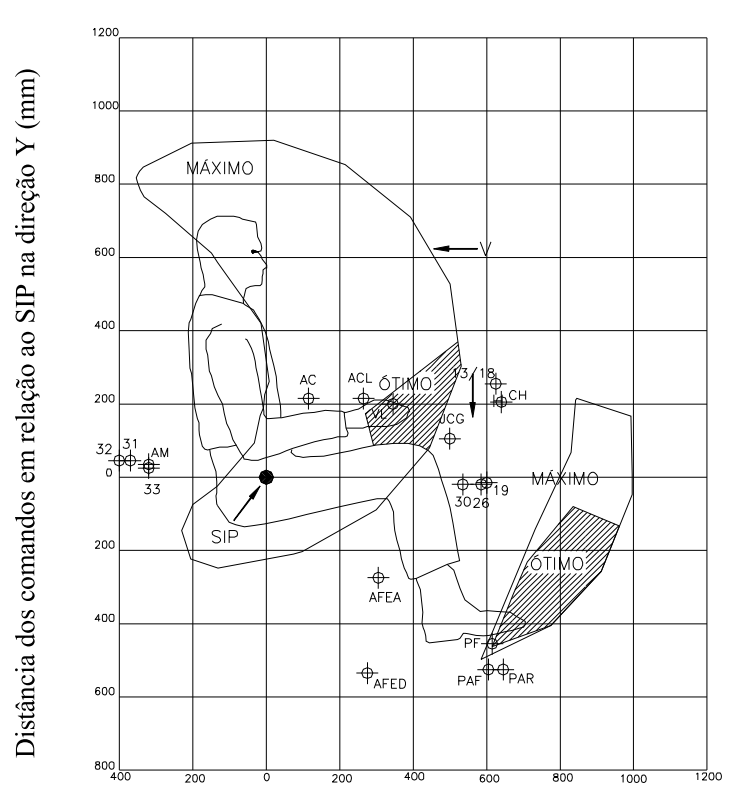

Distância dos comandos em relação ao SIP na direção X (mm) a maior porcentagem dos comandos bem localizados, seguido do "skidder" Caterpillar 545, "forwarders" Timberjack 1210B, Timberjack 1710D e Volvo A25C e, por último, o "skidder" Tigercat 630B.

Quadro 11 - Avaliação da localização dos comandos (\%) com o assento localizado na posição "média"

Table 11 - Evaluation of command position (\%) with the seat in its middle position

\begin{tabular}{|c|c|c|}
\hline \multirow[t]{2}{*}{ Tratores } & \multicolumn{2}{|c|}{ Conceito } \\
\hline & $\mathrm{A}^{(*)}$ & $\mathbf{B}^{(* *)}$ \\
\hline & \multicolumn{2}{|c|}{$\%$} \\
\hline "Forwarder" Valmet 890.2 & 66,7 & 33,3 \\
\hline "Forwarder" Timberjack 1210B & 48,5 & 51,5 \\
\hline "Forwarder" Timberjack 1710D & 43,0 & 57,0 \\
\hline "Forwarder" Volvo A25C & 42,0 & 58,0 \\
\hline "Skidder" Caterpillar 545 & 54,5 & 45,5 \\
\hline "Skidder" Tigercat 630B & 26,0 & 74,0 \\
\hline
\end{tabular}

(*) $\mathrm{A}=$ ("ótimo" + "muito bom" + "bom").

$(* *) \mathrm{B}=$ ("regular" + "ruim").

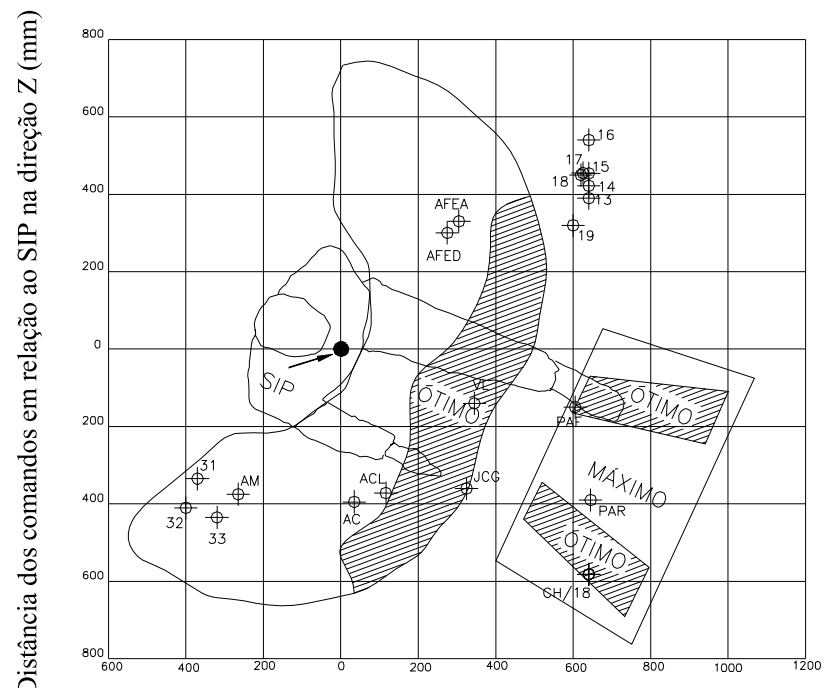

Distância dos comandos em relação ao SIP na direção X (mm)

1- Luz do Nível de Óleo Hidráulico Baixo; 2 - Luz de Temperatura de Arrefecimento; 3 - Luz da Pressão Óleo Motor; 4 - Luz da válvula de descarga de bomba principal; 5 - Luz do Freio de Estacionamento; 6 - Luz de Temperatura Óleo Hidráulico; 7 - Luz de Temperatura Arrefecimento Baixa; 8 - Luz do Filtro de Ar; 9 - Luz do Filtro Hidrostático; 10 - Luz da Pressão da Garra; 11 - Luz Trava do Diferencial Dianteiro; 12 - Luz Trava do Diferencial Traseiro; 13 - Inter. Luzes Frente; 14 - Inter. Luzes Traseiras; 15 - Inter. Luz de Serviço Motor; 16 - Inter. Válvula Descarga Bomba Principal; 17 - Inter. Ignição (Chave); 18 - Inter. Teste do Bulbo; 19 - Acendedor de Cigarro; 20 - Relógio Temperatura de Resfriamento; 21 - Relógio de Pressão do Óleo Motor; 22 - Relógio Temperatura Óleo Hidráulico; 23 - Relógio Nível de Combustível; 24 - Relógio de Amperagem; 25 - Relógio do Horímetro; 26 - Relógio de RPM; PAF - Pedal do Acelerador Frente; PAR - Pedal do Acelerador Ré; 30 - Volante; JCG - Joystick do Controle da Garra; ACL - Alavanca de Controle Lâmina; AC - Alavanca de Cambio; AM - Acelerador Manual; 31 - Inter. Pos. Rotativa de Controle Ar/Ventilador; 32 - Inter. Controle Temperatura; 33 - Inter. Posição de Circulação ar; AFEA - Alavanca Freio Estacionamento (Acionada); AFED - Alavanca Freio de Estacionamento (Desacionada).

Figura 7 - Localização dos órgãos de comandos do "skidder" Tigercat 630B, nas três dimensões.

Figure 7 - Command position of Tigercat 630B, in three dimensions.

R. Árvore, Viçosa-MG, v.31, n.1, p.71-81, 2007 
Pôde-se observar ainda que, em todos os tratores avaliados, os comandos que se encontravam localizados em posições "regular" e "ruim" eram, na maioria das vezes, interruptores de farol de trabalho, do arcondicionado e limpador de pára-brisa, entre outros, geralmente pouco acionados durante a jornada de trabalho.

\section{CONCLUSÃO}

A melhor máquina avaliada quanto ao posicionamento dos comandos, de acordo com o biótipo do conjunto de operadores brasileiros considerados neste estudo, foi o "forwarder" Valmet 890.2, seguido do "skidder" Caterpillar 545, sendo os únicos tratores que apresentaram mais da metade dos comandos bem posicionados, 66,7 e 54,5\%, respectivamente. Nesse caso, o conceito encontrado na bibliografia, quanto ao melhor projeto ergonômico do "forwarder" em relação ao "skidder", não se comprovou em relação a todos os modelos aqui considerados. Conclui-se, finalmente, que há a necessidade de análise do posto de trabalho das máquinas florestais importadas, com relação à sua adaptação ergonômica ao biotipo do trabalhador florestal brasileiro.

\section{AGRADECIMENTOS}

À Fundação de Amparo à Pesquisa do Estado de São Paulo (FAPESP), pelo financiamento do projeto.

\section{REFERÊNCIAS}

\section{ASSOCIAÇÃO BRASILEIRA DE NORMAS} TÉCNICAS - ABNT. Ergonomia. Disponível em:<http://www.abergo.org.br>. Acesso em: 20 jun. 2003.

\section{ASSOCIAÇÃO BRASILEIRA DE NORMAS} TÉCNICAS - ABNT. NBR NM-ISO 5353: Máquinas rodoviárias, tratores e máquinas agrícolas e florestais - ponto de referência do assento. Rio de Janeiro: 1999. 5p.
FOntAnA, G. Avaliação ergonômica do projeto interno de cabines de "Forwarders" e "Skidders". 2005. 85 f. Dissertação (Mestrado em Agronomia) - Escola Superior de Agricultura Luiz de Queiroz, Piracicaba, 2005.

FONTANA, G.; SEIXAS, F. Levantamento antropométrico de operadores brasileiros de tratores florestais "Forwarders" e "Skidders". Revista Engenharia Rural, v.17, n.1, p.41-46, 2006.

LIMA, J.S.S. et al. Avaliação de alguns fatores ergonômicos nos tratores "Fellerbuncher" e "Skidder" utilizados na colheita de madeira. Revista Árvore, v.29, n.2, p.291-298, 2005.

MAKKONEN, I. Choosing a wheeled shortwood forwarder. (FERIC Technical Note, 136), Quebec: Feric, 1989. 12p.

MINETTE, L.J. et al. Avaliação dos efeitos do ruído e da vibração no corte florestal com motoserra. Revista Árvore, v.22, n.3, p.325-330, 1998.

SILVA, C.B.; SANT’ ANNA, C.M.; MINETTE, L.J. Avaliação ergonômica do "feller-buncher" utilizado na colheita de eucalipto. CERNE, v.9, n.1, p.122-131, 2003.

\section{SKOGFORSK - THE FORESTRY RESEARCH INSTITUTE OF SWEDEN. Ergonomic guidelines for forest machines.} Uppsala: Swedish National Institute for Working Life, 1999. 86p.

ZANDER, J. Ergonomics in machine desing: a case study of the self-propelled combine harvester. Wageningen: Mededelingen Landboowhoge-school, 1972. 161p. 
